\title{
Vibrational excitation of cyclopropane by electron impact: An experimental test of the discrete-momentum-representation theory with density-functional-theory approximation of polarization and correlation
}

\author{
R. Čurík* and P. Čársky \\ J. Heyrovský Institute of Physical Chemistry, Academy of Sciences of the Czech Republic, v.v.i., Dolejškova 3, \\ 18223 Prague 8, Czech Republic \\ M. Allan \\ Department of Chemistry, University of Fribourg, CH-1700 Fribourg, Switzerland \\ (Received 29 October 2012; published 21 December 2012)
}

\begin{abstract}
The discrete-momentum-representation theory with short-range correlation and polarization approximated by local-density-functional theory has been augmented by connecting the short-range potential to an asymptotic polarization tensor. The capacity of this theory to correctly describe the coupling of a free electron with nuclear motion is tested by a detailed comparison of calculated and measured cross sections for vibrational excitation in cyclopropane. Absolute magnitudes of the cross sections, selectivity with respect to the vibrational modes excited, angular distributions in the entire angular range $0^{\circ}-180^{\circ}$, and resonant structures dependent on the incident electron energy in the range from threshold to $16 \mathrm{eV}$ are compared. The results are encouraging; the theory reproduces all these aspects quantitatively, even at low energies, down to about $1 \mathrm{eV}$. An important asset of this theory is that it is applicable to large molecules.
\end{abstract}

DOI: 10.1103/PhysRevA.86.062709

PACS number(s): $34.80 . \mathrm{Lx}, 34.50 . \mathrm{Ez}$

\section{INTRODUCTION}

Induction of motion of nuclei (vibrational excitation and chemical change) by electron impact plays a key role in plasmas and their numerous technological and natural applications. It is thus desirable to understand these processes and to be able to calculate quantitative (absolute) ab initio cross sections for them. For polyatomic_-in particular, more than triatomic-molecules we are at present far from this goal [1]. This is because the coupling of the transient negative ion (resonance) with the continuum of free electrons precludes the use of standard quantum-chemical methods (into whose development an immense amount of effort has been invested) but requires scattering calculations. There are several standard methods for treating the scattering problem, such as the Schwinger multichannel variational procedure [2-4], the $R$-matrix method [5], the symmetry-adapted single-center expansion approach [6], and the complex Kohn method [7-9], but for larger molecular systems their complexity and enormous computational effort generally limits them to calculations of fixed-nuclei elastic data. (For diatomic and, in rare cases, triatomic molecules, calculations of quantitative cross sections for vibrational excitation and in some cases even dissociative attachment do exist; examples are given in a recent review [10] and Refs. [8], [9], and [11].)

This work reports on an attempt to improve the situation for larger than triatomic molecules. It presents an important improvement of the discrete-momentum-representation (DMR) theory and tests its capability by a rigorous comparison with an experiment for a prototype molecule. The DMR theory has recently been extended to include short-range correlation and polarization approximated by local and nonlocal density-functional-theory (DFT) models [12,13]. This paper connects the short-range anisotropic potential smoothly to a general and anisotropic asymptotic form for inelastic scattering. The theory is then used to calculate cross sections for vibrational excitation in harmonic approximation and the results are compared to experiment. The test is stringent in the sense that quantitative (absolute) values of the cross sections are compared, all vibrational modes are included, and angular distributions over the entire angular range $\left(0^{\circ}-180^{\circ}\right)$ and the energy range from threshold to $16 \mathrm{eV}$ are considered.

A hydrocarbon molecule was chosen as the test case because hydrocarbons are an important class of molecules in plasmas. Plots of vibrational excitation cross sections in saturated hydrocarbons as a function of electron energy generally consist of a single very broad band extending from threshold to above $10 \mathrm{eV}$, within which individual resonances cannot be distinguished and where comparison with calculated cross section would not yield detailed information. Cyclopropane solves this problem because its high symmetry has the consequence of narrowing the resonances by decreasing the contributions of low partial waves, which have low centrifugal barriers and represent a fast channel for the electron to escape. Individual resonances can consequently be distinguished in the spectra $[14,15]$ and permit us to test the capacity of the theory to describe them.

\section{THEORY}

The theoretical approach was described in detail in our recent review [13]. It is a two-channel approach expressed by a pair of coupled Lippmann-Schwinger equations for the transition operator $T$,

$$
\begin{aligned}
& T_{00}=U_{00}+U_{00} G_{0} T_{00}+U_{01} G_{1} T_{10}, \\
& T_{10}=U_{10}+U_{10} G_{0} T_{00}+U_{11} G_{1} T_{10},
\end{aligned}
$$


where the first equation refers to the elastic channel and the second to a selected single vibrational mode. Symbols $G_{0}$ and $G_{1}$ are free-electron Green's functions corresponding to the elastic and inelastic channels, respectively. Since Eqs. (1) and (2) are solved in momentum space the advanced free Green's functions have the following simple form:

$$
\begin{gathered}
G_{0}\left(\vec{k}, \vec{k}^{\prime}\right)=\delta\left(\vec{k}-\vec{k}^{\prime}\right) \int d \vec{k} \frac{1}{E_{0}-k^{2} / 2+i \epsilon}, \\
G_{1}\left(\vec{k}, \vec{k}^{\prime}\right)=\delta\left(\vec{k}-\vec{k}^{\prime}\right) \int d \vec{k} \frac{1}{E_{0}-E_{v}-k^{2} / 2+i \epsilon} .
\end{gathered}
$$

The elastic channel energy is denoted $E_{0}$ and the vibrational energy of the computed vibrational mode $E_{v}$.

The interaction potential $U$ is of the SEP type, consisting of three components: static, exchange, and polarization. The first two are treated rigorously in a mixed plane-wave and Gaussian basis and they are generated from the Hartree-Fock wave function at the equilibrium geometry of the molecule. The polarization potential is approximated by a short-range DFT correlation potential [16] given by the DFT but obtained from the Hartree-Fock electron density. It is smoothly connected [12] to the asymptotic polarization term

$$
V_{p}(\mathbf{r})=-\frac{1}{2 r^{6}} \sum_{i, j}^{3} \alpha_{i j} x_{i} x_{j}
$$

where $\alpha_{i j}$ are components of the dipole polarizability tensor of the target molecule. Different theoretical groups give different meaning to the SEP acronym. In many-electron methods the SEP acronym means polarization effects included by closed-channel single-electron excitations of the target molecule $[17,18]$. In optical potential methods (as in the present work) polarization forces are included in the form of an additional local potential which may or may not be based on a DFT modeling [19,20].

Harmonic approximation is used for the vibrations; interaction between different vibrational modes is therefore neglected, and the $U_{10}$ elements may be obtained simply as derivatives of the $U_{00}$ elements with respect to normal coordinates. The derivatives of correlation, electrostatic, and exchange components can be evaluated analytically [21]. The derivatives of asymptotic polarizability tensor $\alpha_{i j}$ in Eq. (5) were obtained by a numerical differentiation using linear response functions in Kohn-Sham DFT [22] calculations with the B3LYP hybrid functional and Sadlej's polarized valence triple $\zeta$ basis set [23] as implemented in the program Dalton 2.0 [24].

In this paper we improve our description of the polarization term by assuming also derivatives of $\alpha_{i j}$ that have not been taken into account in the model [13] so far. Explicit integration of the $U G T$ terms in Eqs. (1) and (2) is bypassed by numerical quadrature tested for numerical stability.

The differential cross sections $\sigma_{10}$ for the vibrational excitation $1 \leftarrow 0$ are proportional to the squared matrix elements of $T_{10}$ [21,25]. The $\sigma_{10}$ manifold represents the line electron energy loss spectrum (EELS). For comparison with experiment the lines are positioned at experimentally observed frequencies and converted to bands by assuming a Gaussian form. The heights of the bands are set at the values of calculated differential cross sections and the assumed half-width of $15 \mathrm{meV}$ was given by the resolution of the experimental apparatus.

We conclude this theoretical section with a note regarding the symmetry properties of the inelastic wave function. The cyclopropane molecule belongs to the $D_{3 \mathrm{~h}}$ point group. Since the vibrational motion generally lowers the symmetry of the molecule (except for the three full-symmetric modes $v_{1}, v_{2}$, and $v_{3}$ ), the symmetry of the vibrationally inelastic wave function is not conserved during the inelastic collision. Formally, $T_{10}$ in Eq. (2) is not block diagonal because $U_{10}$ has the symmetry of the computed vibrational mode [26] and therefore it may couple different irreducible angular spaces of incoming and outgoing parts of the inelastic electron wave function. In the present computational study we determine the symmetry of the inelastic wave function by inspection of dominant partial waves in incoming and outgoing channels of the $T_{10}$ matrix.

\section{EXPERIMENT}

The earlier measurements of cyclopropane [14,15] were substantially improved and extended for this study. The absolute magnitudes of the cross sections were measured, the resolution was improved, and the angular distribution measurements were extended to $180^{\circ}$ using a magnetic angle changer. Measurements were performed with a spectrometer using hemispherical analyzers [27,28]. Absolute values of the cross sections were determined by the relative flow technique and normalized to a theoretical helium elastic cross section as described earlier. The confidence limit for the magnitudes of the inelastic cross sections is about $\pm 25 \%$ (2 standard deviations). Angular distributions were measured using combined mechanical setting of the analyzer and magnetic deflection using a magnetic angle changer. The resolution was about $15 \mathrm{meV}$ in the energy loss mode.

\section{RESULTS AND DISCUSSION}

In Fig. 1 we present the experimental and calculated electron energy loss spectra for the incident electron energy of $5.5 \mathrm{eV}$ - the center of an observed resonance. An energy loss spectrum provides an overview of the cross sections at one energy and one scattering angle, but for all vibrational modes. Several normal modes overlap with the present instrumental resolution, but comparison of the measured and calculated envelopes still provides a stringent test for all modes. The individual bands in the spectrum, although due to several overlapping modes, often belong to one type of motion. The band at $380 \mathrm{meV}$ is due to various $\mathrm{C}-\mathrm{H}$ stretch modes; the band at $180 \mathrm{meV}$, to $\mathrm{CH}_{2}$ scissoring modes; the band at $145 \mathrm{meV}$ to the $\mathrm{C}-\mathrm{C}$ ring stretch mode $v_{3}\left(a_{1}^{\prime}\right.$, at $\left.147 \mathrm{meV}\right)$, overlapping with the two $\mathrm{CH}_{2}$ twist modes $v_{4}\left(a_{1}^{\prime \prime}\right.$, at $\left.139 \mathrm{meV}\right)$ and $v_{13}\left(e^{\prime \prime}\right.$, at $147 \mathrm{meV})$; and the band at $107 \mathrm{meV}$, to the ring deformation mode $v_{11}\left(e^{\prime}\right.$, at $\left.107 \mathrm{meV}\right)$, overlapping with the $\mathrm{CH}_{2}$ rocking mode $v_{7}\left(a_{2}^{\prime \prime}\right.$, at $\left.106 \mathrm{meV}\right)$. The shoulder at $92 \mathrm{meV}$ is due to the $\mathrm{CH}_{2}$ rocking mode $v_{14}\left(e^{\prime \prime}\right)$. Agreement of theory with experiment is excellent, in part doubtlessly fortuitous in view of our instrumental confidence limit of $25 \%$.

Earlier experimental studies [14,15] revealed a relatively narrow resonance at $5.5 \mathrm{eV}$ in the cross section for exciting the $\mathrm{C}-\mathrm{C}$ ring stretching mode $v_{3}$. Qualitative interpretation of the 


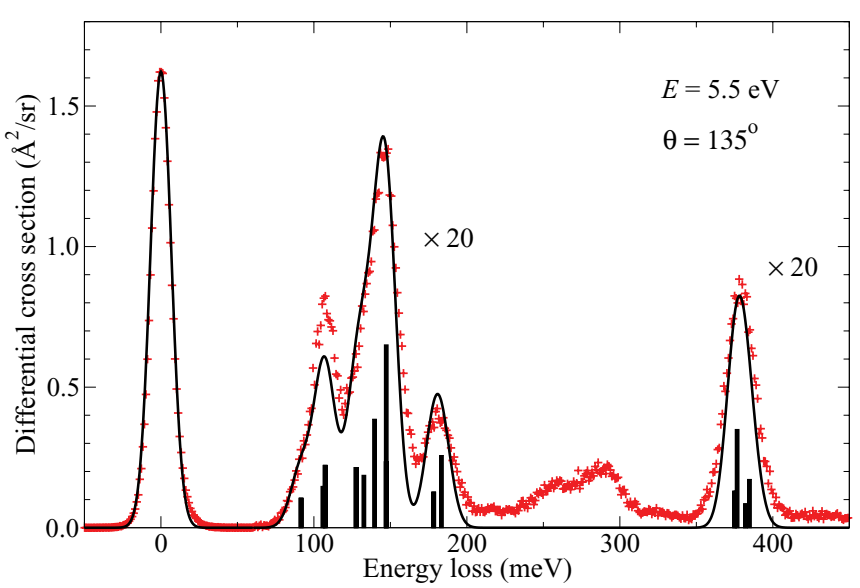

FIG. 1. (Color online) Electron energy loss spectrum of cyclopropane for an electron incident energy of $5.5 \mathrm{eV}$ and a scattering angle of $135^{\circ}$. Experimental EELS is denoted by crosses, while the solid line is the calculated DMR SEP spectrum. Both experimental and theoretical data are plotted on an absolute scale. The signal in the 200- to $350-\mathrm{meV}$ range of the experimental curve is due to overtones and combination bands that are not amenable to treatment in harmonic approximation.

angular distribution and mode selectivity led to its assignment as a temporary electron capture to a $\sigma_{\mathrm{C}-\mathrm{C}}^{*}$ orbital antibonding with respect to all C-C bonds, with $A_{2}^{\prime}$ symmetry.

The present computation permits a much deeper insight into the excitation mechanism. It confirms that the inelastic scattering wave function for the $v_{3}$ mode consists almost exclusively of a single partial wave $l=3, m=3$, which is the lowest partial wave of $A_{2}^{\prime}$ symmetry (with a carbon ring in the $x y$ plane and one carbon atom on the $y$ axis). The $f$-wave shape of $v_{3}$ excitation is very clearly demonstrated in the angular dependence shown in Fig. 2.

However, the excitation of vibrational mode $v_{3}$ alone is not sufficient to explain quantitatively the measured angular dependence: the experimental undulations in the angular

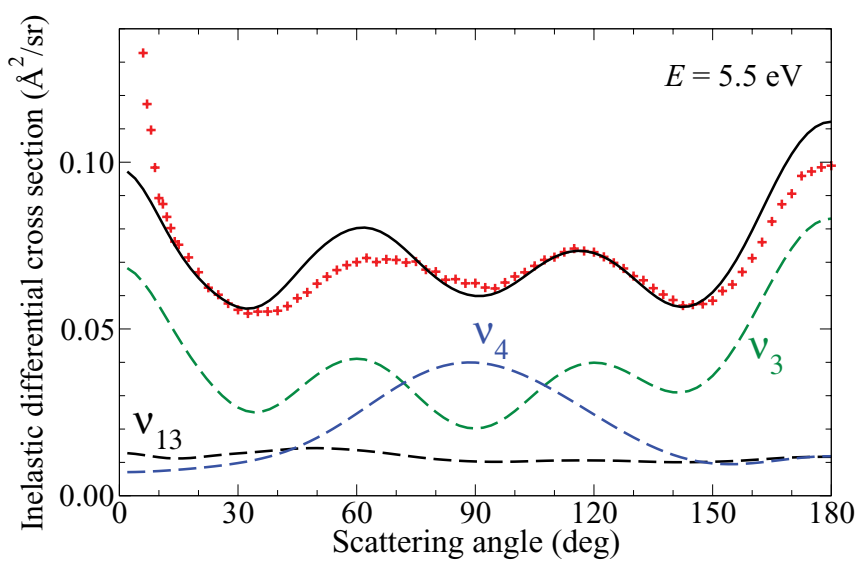

FIG. 2. (Color online) Differential cross section for the energy loss of $147 \mathrm{meV}$, the nominal energy of the $\mathrm{C}-\mathrm{C}$ ring stretch vibration $v_{3}$. Experimental data are shown by crosses. The signal expected at $147 \mathrm{meV}$, resulting from partial band overlap and calculated as a weighted sum assuming $15-\mathrm{meV}$ Gaussian profiles, is denoted by the solid curve.

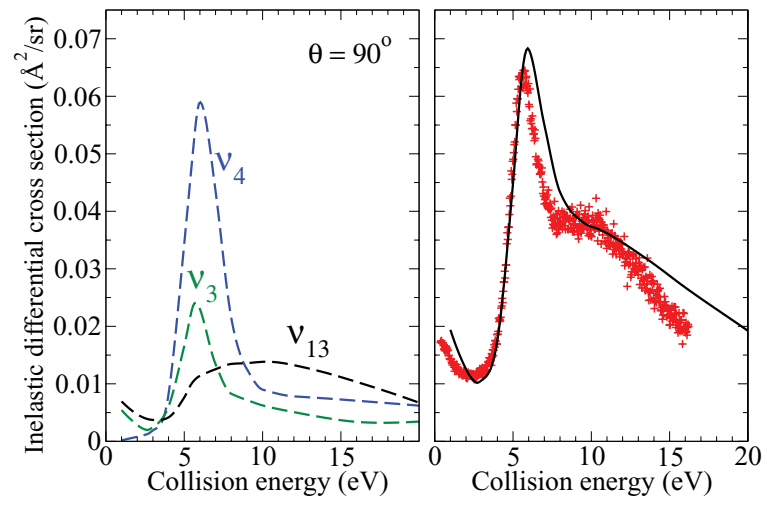

FIG. 3. (Color online) Left: Differential cross sections for vibrational excitation for the $v_{3}, v_{4}$, and $v_{13}$ modes of cyclopropane calculated as a function of the collision energy. Right: Sum of three contributions with the assumed half-width of $15 \mathrm{meV}$ (solid curve) compared with experiment (crosses).

distribution are less deep than expected for a pure $f$ wave. We note that also the $\mathrm{HCH}$ twisting modes $v_{13}(147 \mathrm{meV}$, doubly degenerate) and $v_{4}(139 \mathrm{meV})$ are in the range of $15 \mathrm{meV}$ of the experimental resolution and hence should be taken into account. The angular dependence of the differential cross sections for the three contributing modes is shown in Fig. 2. Since the $v_{4}$ mode lies a little aside the measured energy loss we estimated its contribution to the measured electron flux to be about $55 \%$ of its calculated cross section. Once the contributions of the three modes are summed up (the contribution of $v_{13}$ being taken twice because of the degeneracy), as shown in Fig. 2, the angular shape at 5.5-eV resonance is well reproduced.

Figure 2 thus shows that the measured angular shape at the $v_{3}$ energy loss of $147 \mathrm{meV}$ and the collision energy of $5.5 \mathrm{eV}$ is influenced by a partial contribution of the $p$-wave shape coming from excitation of the $v_{4}$ mode. In Fig. 3 we demonstrate that this contribution is also resonant. Our calculations show unambiguously that in this case the $A_{2}^{\prime}$ resonance leaks out as $A_{2}^{\prime \prime}$ with the lowest partial wave $l=1$, $m=0$, which is consistent with the angular shape of $v_{4}$ excitation found in Fig. 2.

A resonance at $2.6 \mathrm{eV}$ was weakly visible in the early experimental data $[14,15]$ for the excitation of the symmetric $\mathrm{HCH}$ scissoring mode $\nu_{2}$ with an energy loss of $183 \mathrm{meV}$. Based on the argument of the antibonding properties of the virtual orbital, the authors have chosen a possible resonance symmetry candidate as $A_{2}^{\prime \prime}$. Our computations show the resonance more pronounced (see Fig. 4) in excitation of $v_{2}$, and indeed the dominant contribution to the cross section comes from $A_{2}^{\prime \prime}$ in both incoming and outgoing channels. This assignment resolves the ambiguity in Ref. [11]. A comparison with the absolute experimental data in Fig. 4 is again not straightforward due to the near-lying asymmetric $\mathrm{HCH}$ scissoring mode $v_{9}$ with an energy loss of $178 \mathrm{meV}$. The estimated band overlap of this doubly degenerate excitation to the measured differential cross section is $72 \%$ of its full intensity. The contribution is also multiplied by factor of 2 because of the degeneracy. The agreement of computed and measured data in Fig. 4 is extraordinarily good. 


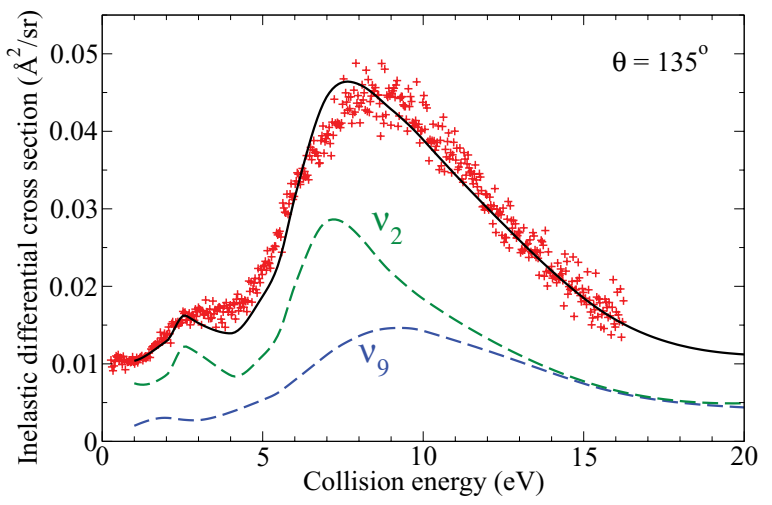

FIG. 4. (Color online) Differential cross section for the energy loss of $183 \mathrm{meV}$ as a function of the collision energy. The weighted sum of the two contributing vibrational modes (solid curve) is compared with the present experimental data (crosses).

\section{CONCLUSIONS}

Comparison with experiment revealed that the present form of the DMR SEP theory is capable of predicting quantitatively vibrational excitation cross sections and thus describing correctly the electron-nuclei coupling in electron-molecule collisions. In particular, the theory correctly describes the resonances at 2.6 and $5.5 \mathrm{eV}$. Their assignment is in agreement with that originally proposed experimentally on the basis of qualitative interpretation of the angular distributions and the selectivity in terms of the modes excited. Our study confirms that the totally symmetric $\mathrm{C}-\mathrm{C}$ ring stretching mode $v_{3}$ is driven by the $5.5-\mathrm{eV}$ shape resonance of $A_{2}^{\prime}$ symmetry with $f$-wave character. The theory reveals aspects of the nuclear dynamics which could not be derived from the experiment alone, however. Thus, we found that the $5.5-\mathrm{eV}$ resonance also decays into $A_{2}^{\prime \prime}$ symmetry via resonant excitation of the nontotally symmetric $\left(a_{1}^{\prime \prime}\right) \mathrm{HCH}$ twisting mode $v_{4}$ (which partially overlaps with $v_{3}$ in the experiment). In this case the angular shape has a strong $p$-wave behavior. This initially unexpected process whereby the outgoing wave has a symmetry different from that of the incoming wave by simultaneous excitation of a nontotally symmetric vibration is even stronger at $90^{\circ}$ than the expected excitation of the totally symmetric $v_{3}$ mode. One may look at it as a manifestation of vibronic coupling.

The inclusion of the advanced approximations for polarizability and correlation thus dramatically improved the capacity of the DMR theory. It is now capable of describing resonances, despite the fact that resonances are not a priori included in the model and were not reproduced by earlier versions of the DMR theory. The resonance energies are reproduced with a remarkable accuracy (errors in resonance energy far exceeding $1 \mathrm{eV}$ are common for scattering calculations). The low-energy capability of the theory is dramatically improved; it extends down to $1-2 \mathrm{eV}$, a remarkable success for an ab initio scattering theory. Combined with the present capacity of this theory to treat systems up to at least 10 heavy atoms, this qualifies it as a promising candidate for quantitative ab initio description of vibrational excitation and electron-driven chemistry of large polyatomic molecules.

\section{ACKNOWLEDGMENTS}

Calculations of polarizabilities $\alpha_{i j}$ in Eq. (5) were performed by I. Paidarová. Her assistance is gratefuly acknowledged. This work was supported in part by COST Action CM0805, the Czech Ministry of Education (Grant No. OC10046) and the Grant Agency of the Czech Republic (Grant No. P208/11/0452). It is also part of Project No. 200020-131962/1 of the Swiss National Science Foundation, of Project SBF No. C07.0018 of the State Secretariat for Education and Research, and of COST Action CM0601.
[1] Y. Itikawa, J. Phys. B 37, R1 (2004).

[2] P. Palihawadana, J. Sullivan, M. Brunger, C. Winstead, V. McKoy, G. Garcia, F. Blanco, and S. Buckman, Phys. Rev. A 84, 062702 (2011).

[3] M. Allan, C. Winstead, and V. McKoy, Phys. Rev. A 83, 062703 (2011).

[4] M. A. Khakoo, J. Muse, K. Ralphs, R. F. da Costa, M. H. F. Bettega, and M. A. P. Lima, Phys. Rev. A 81, 062716 (2010).

[5] Z. Mašín and J. D. Gorfinkiel, J. Chem. Phys. 135, 144308 (2011).

[6] I. Baccarelli, I. Bald, F. A. Gianturco, E. Illenberger, and J. Kopyra, Phys. Rep. 508, 1 (2011).

[7] C. S. Trevisan, A. E. Orel, and T. N. Rescigno, Phys. Rev. A 74, 042716 (2006).

[8] D. J. Haxton, Z. Zhang, H. D. Meyer, T. N. Rescigno, and C. W. McCurdy, Phys. Rev. A 69, 062714 (2004).

[9] D. S. Slaughter, H. Adaniya, T. N. Rescigno, D. J. Haxton, A. E. Orel, C. W. McCurdy, and A. Belkacem, J. Phys. B 44, 205203 (2011).
[10] P. Čársky and R. Čurík (eds.), Low-Energy Electron Scattering from Molecules, Biomolecules and Surfaces (CRC Press, Boca Raton, FL, 2012).

[11] R. Čurík and F. A. Gianturco, J. Phys. B: At. Mol. Opt. Phys. 35, 1235 (2002).

[12] R. Čurík and M. Šulc, J. Phys. B 43, 175205 (2010).

[13] P. Čársky and R. Čurík, in Low-Energy Electron Scattering from Molecules, Biomolecules and Surfaces, edited by P. Čársky and R. Čurík (CRC Press, Boca Raton, FL, 2012).

[14] M. Allan, J. Am. Chem. Soc. 115, 6418 (1993).

[15] M. Allan and L. Andric, J. Chem. Phys. 105, 3559 (1996).

[16] N. T. Padial and D. W. Norcross, Phys. Rev. A 29, 1742 (1984).

[17] T. Beyer, B. Nestmann, and S. Peyerimhoff, Chem. Phys. 255, 1 (2000).

[18] C. Winstead, V. McKoy, and M. H. F. Bettega, Phys. Rev. A 72, 042721 (2005).

[19] M. A. Morrison and L. A. Collins, Phys. Rev. A 17, 918 (1978).

[20] S. Telega, E. Bodo, and F. A. Gianturco, Eur. Phys. J. D 29, 357 (2004). 
[21] R. Čurík and P. Čársky, J. Phys. B 36, 2165 (2003).

[22] Z. Rinkevicius, I. Tunnel, P. Salek, O. Vahtras, and A. Hans, J. Chem. Phys. 119, 34 (2003).

[23] J. Sadlej, Coll. Czech. Chem. Commun. 53, 1955 (1988).

[24] DALTON, a Molecular Electronic Structure Program, Release 2.0 (2005), http://www.kjemi.uio.no/software/dalton/ dalton.html.
[25] F. A. Gianturco, D. G. Thompson, and A. Jain, in Computational Methods for Electron-Molecule Collisions, edited by W. M. Huo and F. A. Gianturco (Plenum Press, New York, 1995).

[26] G. A. Gallup, J. Chem. Phys. 99, 827 (1993).

[27] M. Allan, J. Phys. B 40, 3531 (2007).

[28] M. Allan, Phys. Rev. A 81, 042706 (2010). 